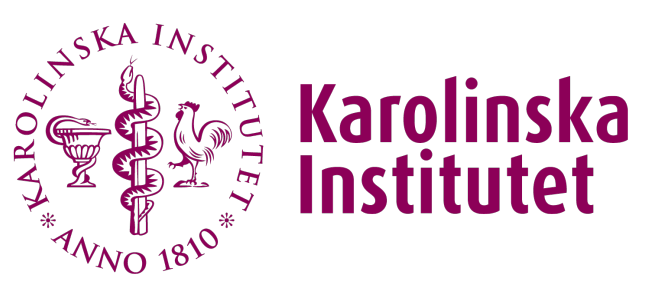

Karolinska Institutet

http://openarchive.ki.se

This is a Peer Reviewed Accepted version of the following article, accepted for publication in Aging and Mental Health.

\title{
Using a screening tool to evaluate potential use of e-health services for older people with and without cognitive impairme
}

Malinowsky, Camilla; Nygård, Louise; Kottorp, Anders

Aging Ment Health. 2014;18(3):340-5.

http://doi.org/10.1080/13607863.2013.832731

http://hdl.handle.net/10616/45001

If not otherwise stated by the Publisher's Terms and conditions, the manuscript is deposited under the terms of the Creative Commons Attribution-NonCommercial-NoDerivatives License (http://creativecommons.org/licenses/by-nc-nd/4.0/), which permits non-commercial re-use, distribution, and reproduction in any medium, provided the original work is properly cited, and is not altered, transformed, or built upon in any way. 


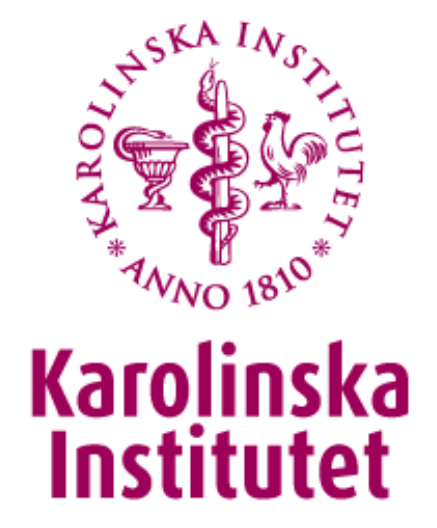

2016-01-18

This is an author produced version of a paper accepted by Aging and Mental Health. This paper has been peerreviewed but does not include the final publisher proofcorrections or journal pagination.

Using a screening tool to evaluate potential use of ehealth services for older people with and without cognitive impairment.

Malinowsky C, Nygård L, Kottorp A.

DOI: $10.1080 / 13607863.2013 .832731$

Access to the published version may require subscription. Published with permission from: Taylor \& Francis 
USING A SCREENING TOOL TO EVALUATE POTENTIAL USE OF E-HEALTH SERVICES FOR OLDER PEOPLE WITH AND WITHOUT COGNITIVE IMPAIRMENT ABSTRACT

Objectives: E-health services are increasingly offered to provide clients with information and a link to healthcare services. The aim of this study is to investigate the perceived access to and the potential to use technologies important for e-health services among older adults with MCI or mild AD and controls. Method: The perceived access to and perception of difficulty in the use of everyday technology (such as cell phones, coffee machines, computers) was investigated in a sample of older adults $(n=118)$ comprising three subsamples: adults with mild cognitive impairment (MCI) $(n=37)$, with mild Alzheimer's disease (AD) $(n=37)$ and controls ( $\mathrm{n}=44)$ using the Everyday Technology Use Questionnaire (ETUQ). The use of seven technologies important for e-health services was specifically examined for each subsample and compared between the subsamples. Results: The findings demonstrated that the older adults in all subsamples both perceive access to e-health technologies and potentially would use them competently in several e-health services. However, among persons with AD a lower proportion of perceived access to the technology was described, as well as for persons with MCI. Conclusion: To make the benefits of e-health services available and used by all clients, it is important to consider access to the technology required in e-health services and also to support the clients' capabilities to understand and use the technologies. Also, the potential use of the ETUQ to explore the perceived access to and competence in using e-health technologies is a vital issue in the use of e-health services.

KEY WORDS: Alzheimer's disease, mild cognitive impairment, healthcare, everyday technology 


\section{INTRODUCTION}

Within the field of healthcare there is a growing emphasis on e-health services to provide clients with easier access to information and self-management. Examples of e-health services are online health guides, e-prescriptions (Jung \& Loria, 2010), and disease management support (Hall, Stellefson \& Bernhardt, 2012). Technological artefacts and services such as the Internet (Jung \& Loria, 2010; Vanagas \& Klimavičiūtè-Gudauskiene, 2012; Van Deursen, 2012) and cell phones (Hall et al., 2012), hereafter named e-health technologies, are central in the use of e-health services. But it may be important to keep in mind that the technology in itself might imply hindrances for certain people in health promotion. Basic prerequisites for use of e-health services are the clients' access to technologies needed and the use of them. It is reported that $94 \%$ of the Swedish population between 16 and 74 years of age have access to a computer and the Internet at home and that $80 \%$ use the Internet daily. However, among older people (65-84 years), a lower rate of access and use is reported (Statistics Sweden, 2013). In addition, in Sweden six out of ten persons between 16 and 74 years have used the Internet in a smart phone/cell phone (Statistics Sweden, 2013). However, to possess or to have access to the technology does not automatically mean that the technology is used competently. The benefits of e-health services will only be attained if the abilities and needs of the users are accounted for in the design of the applications (Czaja et al., 2012). This is specifically important for older adults experiencing functional decline or disabilities (Atkinson et al., 2011). Earlier studies have demonstrated that older adults with cognitive impairment caused by Alzheimer's disease (AD) or mild cognitive impairment (MCI) perceive more technological difficulties in everyday life compared to those with no known cognitive impairment on a group level (Nygård, Pantzar, Uppgard \& Kottorp, 2011; Rosenberg, Kottorp, Winblad \& Nygård, 2009a). 
Among older adults in general, the need for contact with healthcare services is often substantial. As e-health services are increasingly used in healthcare services and older adults may have difficulties using them, it is important to investigate their potential to use e-health services more empirically. The aim of this study is therefore to investigate in more depth the perceived access to and potential to use technologies important for e-health services among older adults with MCI or mild AD and controls.

\section{METHODS AND MATERIAL}

Data for this study originate from a secondary analysis of earlier findings regarding the perceived difficulty in technology used in everyday life in a sample of older adults $(n=118)$ comprising three subsamples. Persons with mild AD, MCI and controls were investigated and studied using the Everyday Technology Use Questionnaire, ETUQ (Nygård et al., 2011; Rosenberg, Nygård, Kottorp, 2009b). In this study, the perceived access to and potential to use technologies in the ETUQ, important for e-health services, were specifically examined.

\section{Participants}

Participants with MCI and AD were recruited through a university hospital unit for early investigation of memory disorders. For MCI, criteria based on Petersen (2004) and Winblad et al. (2004) had to be met. Participants with AD had been diagnosed based on NINCDSADRDA (McKhann et al., 1984) and DSM-IV (APA, 2000). If other diagnoses that could cause cognitive impairments such as stroke or depression were present, potential participants were not included. The older adults with no known cognitive impairment, termed controls, were recruited through voluntary retirement organizations. The demographics of the samples are presented in Table 1. 


\section{Instruments}

The ETUQ was developed to identify peoples' perceptions of difficulty in using the everyday technology (ET) that they perceived access to, such as computers, automatic telephone services, stereos and microwaves (Nygård et al., 2011; Rosenberg et al., 2009b). The ETUQ has demonstrated acceptable psychometric properties in earlier studies of adults with and without cognitive impairments (Nygård et al., 2011; Rosenberg, 2009a; 2009b). The ETUQ comprises 92 items, i.e., technological artefacts and services, and it is administered in 30-45 minutes as a face-to-face interview. A six-step rank-category scale is used to register answers (see Table 2). Earlier findings have demonstrated that some of the scale steps cannot be differentiated from each other statistically upon the clients' responses and therefore are collapsed in the following analysis as recommended in the literature (Linacre, 2004). In the previous rating scale analysis (Nygård et al., 2011), the A and B as well as the C and D categories were disordered and they were therefore collapsed as A/B and C/D (Linacre, 2004). In the present analysis, the categories in the ETUQ were dichotomized into two categories "Used with frequent/major difficulties or sometimes together with another person" and "Independent or with minor difficulties" in order to distinguish persons" potential to competently use the e-health technologies from those who potentially could not.

\section{Insert Table 2 about here}

\section{Data-gathering procedures}

Four experienced and specially trained research assistants collected all data, most often in each participant's home following the standardized procedure (Nygård, 2008; Nygård et al., 2011; Rosenberg et al., 2009b). Based on our earlier experiences (Rosenberg et al., 2009a; 2009b), some participants preferred to include a significant other as support in the standardized interview because this had been shown to facilitate the interview (see Table 1). 
Before the study was initiated, an approval from the Regional Ethical Committee was obtained (D-n [journal number]: 2008/304-31/2).

\section{Data analysis}

In order to explore the proportion of perceived access to and difficulties using e-health technologies among people with $\mathrm{MCI}, \mathrm{AD}$ and controls, the analysis was performed in several steps. Firstly, among the 92 technologies in the ETUQ, seven potential e-health technologies based upon empirical experience and literature were selected (Table 3). The proportion in percentage of persons currently perceiving access to these seven technologies was then calculated for each of the subsamples (Table 3). Secondly, the responses in the ETUQ were analyzed using a Rasch model, using the WINSTEPS computer software program version 3.69.1.16 (Linacre, 2009). The criterion for acceptable goodness-of-fit for item (ET) was set as infit $M n S q$ values below 1.4 (Bond \& Fox, 2007). If this criterion was met, the included ehealth technologies would be considered to fit with other ETs in the ETUQ and further support evidence of unidimensionality of the scale (i.e., all technologies support a single underlying construct). The measures generated from the WINSTEPS program could be used as valid measures for further statistical analysis. From the analysis, each of the seven e-health technologies also received a measure of perceived level of difficulty, and each of the 118 participants received a measure of perceived difficulty in ET use. This process has been described in detail elsewhere (Nygård et al., 2011; Rosenberg et al., 2009b).Thirdly, based upon the rating scale used in the ETUQ (Table 4) and earlier findings of rating scale functioning in this sample (Nygård et al., 2011), we decided to analyze the level of difficulty for e-health technologies in relation to the sample person's measures by placing the technologies along the scale where there was a 50/50 probability of receiving a score for using the specific ET: Independently or with minor difficulties versus with frequent/major 
difficulties or sometimes together with another person. Finally, the Statistical Package for Social Sciences (SPSS) version 20 (2011) was used in order to further explore patterns among subsamples. The subsamples were analyzed with Fisher's exact tests to investigate whether they passed/did not pass the e-health technologies' cutoffs with a level of significance set at $p<0.05$.

\section{Insert Table 3 about here}

\section{RESULTS}

In the analysis of the seven e-health technologies, all demonstrated acceptable goodness-of-fit to the Rasch model according to the criteria set. The levels of perceived difficulty for the ehealth technologies were also equally spread among the more challenging ETs of the 92 in the ETUQ.

The calculations of proportions of persons in the subsamples perceiving access to the e-health technologies (Table 3) showed that among controls and persons with MCI, two thirds or more of the participants perceived access to all of the e-health technologies. Even among persons with mild $\mathrm{AD}$, the perceived access to the technologies ranged from $30 \%$ to $97 \%$. In Figure 1 the distribution of participants' measures of perceived difficulty in ET use in the subsamples are placed parallel to the level of perceived difficulty for the seven e-health technologies. The potentials to use the e-health technologies are presented for each of the subsamples in Table 4. Controls had the highest potential to competently use the e-health technologies, followed by persons with $\mathrm{MCI}$ and persons with $\mathrm{AD}$. It was also demonstrated that the potential to use the seven e-health technologies competently differed significantly between the subsamples with $\mathrm{AD}$-controls $(p<.001)$ and AD-MCI $(p<.001, p=0.005, p=0.005, p=0.005, p=0.005, p=0.014$, 
$p=0.014$, while the differences between MCI-controls only differed significantly concerning the use of a cell phone: text message $(p=0.015)$.

\section{Insert Figure 1 about here \\ Insert Table 4 about here}

\section{DISCUSSION}

In this study, it was empirically demonstrated that older adults with and without cognitive impairment both perceive access to e-health technologies, and individuals in all groups would potentially manage to use these in several e-health services. However, among persons with mild AD or MCI, potential limitations and difficulties were identified. In order to make the benefits of e-health services available and used by all clients, it is of importance to consider and support the clients' access and abilities to understand and use the technologies required in e-health services (Manafo \& Wong, 2012; US Department of Health and Human Services, 2006). Interventions that could compensate for cognitive declines such as making websites easier to comprehend and providing tools to support internet use, have already been suggested to enable people with cognitive impairments to successfully use e-health technologies in relation to healthcare services (Czaja et al. (2012). Such interventions are empirically supported by the findings in this study specifically, as, many persons with AD were demonstrating difficulties in the use of several e-health technologies.

These findings also to some extent support other studies of e-health services among the elderly (Czaja et al., 2012; Pak, Price \& Thatcher, 2009). But the findings here also reveal new information of clinical importance for healthcare. Firstly, they show that we cannot assume that people with MCI or AD as groups will automatically be non-users of e-health services due to their diagnoses. The level of perceived difficulty among e-health technologies 
indicates that several persons with MCI and AD will be potentially able to use some of them, e.g. search information on the Internet "with only minor or no perceived difficulties." None of the included e-health technologies were in all cases assessed to be used "with frequent/major difficulties or sometimes together with another person" for the persons with MCI and AD. These findings suggest that predicting success must be judged on an individual level, as the variations in the groups are large. Secondly, the levels of perceived difficulty calibration measures of a variety of everyday technologies, including e-health technologies, in combination with the individual's generated measure can be used as an empirical guide in the ETUQ to evaluate each individual's potential to use such technologies (Nygård et al., 2011; Rosenberg, 2009a; 2009b). Also, this study supports the potential use of the ETUQ to explore the specific perceived access to and competence in using e-health technologies. Finally, the ehealth technologies' levels of difficulty and relative positions in the continuum are based upon peoples' actual perceived difficulties when using them. For e-health technologies not yet accessible to and used by the client group, we may expect even more people experiencing major problems when initiating the use of e-health services (Vanagas \& KlimavičiūtèGudauskiene, 2012), as they would be unfamiliar until integrated into his/her everyday life and habits.

This is a pilot study. In future studies more e-health technologies and larger samples need to be included in order to monitor levels of difficulty and a person's ability as well as to suggest different strategies. In this study, the sample consists only of 118 persons divided into three subsamples. Potential participants with $\mathrm{AD}$ and $\mathrm{MCI}$ were not randomized into the study but were identified and invited to participate based on stated inclusion- and exclusion criteria by professionals at a unit for early investigation of memory disorders in collaboration with members of the research group. Controls were recruited through voluntary retirement 
organizations. There is certainly a risk that this sample is not representative of all older adults with and without cognitive impairment. Also, the use of e-health technologies needs to be assessed in the context of e-health services. In the present study, the perceived level of difficulty for the technologies was assessed for general use, such as computer use to search for information on the Internet, and not specifically focused on information related to e-health services. Lastly, the increasingly faster changes in trends of technology use also need to be considered in the clinical evaluations used to monitor how well people manage such technologies. However, one of the major advantages of ETUQ is that it is based on a Rasch model, and the generated measures are based upon the pattern of responses rather than on the sum of raw scores, as in most traditional assessments. This allows us to continuously add new and relevant technologies (including e-health services) to the ETUQ item bank and, as long as the added technologies fit the Rasch model assertions, they can be used within the evaluation and still generate comparable measures across people. The development of new technologies such as smart phones, touchpads and touch computers will of course affect everyone's management of technology in everyday life over time and this fact calls for further studies, especially to explore the consequences among people with cognitive impairments when interacting with health care services.

\section{CONFLICT OF INTEREST}

None. 
First, the authors thank the respondents for participating in the study. Thanks also to occupational therapists Monica Pantzar, Sofia Starkhammar, Cecilia Toding and Brittmari Uppgard who took part in the data gathering. This research was financially supported by Swedish Brainpower, the regional agreement on medical training and clinical research between the Stockholm County Council and the Karolinska Institutet and Strategic Research Programme in Care Sciences.

\section{REFERENCES}

American Psychiatric Association (APA). (2000). Diagnostic and Statistical Manual of Mental Disorders. $4^{\text {th }}$ ed. (DSM-IV), Washington, DC: Author.

Atkinson, N. L., Massett, H. A., Mylks, C., McCormack, L. A., Kish-Doto, J., \& Hesse, B. W. (2011). Assessing the impact of user-centered research on a clinical trial eHealth tool via counterbalanced research design. Journal of American Medical Informatics Association. 18, 24-31.

Bond, T. G. \& Fox, C. M. (2007). Applying the Rasch model: Fundamental measurement in the human sciences. Mahwah, NJ: Lawrence Erlbaum.

Czaja, S. J., Sharit, J., Lee, C. C., Nair, S. S. Hernández, M. A., Arana, N., et al. (2012). Factors influencing use of an e-health website in a community sample of older people. Journal of American Medical Informatics Association. DOI: 10.1136/amiajnl-2012-000876

Hall, A. K., Stellefson, M., \& Bernhardt, J. M. (2012). Healthy Aging 2.0: the potential of new media and technology. Preventing Chronic Disease, 9, 110241. DOI: http://dx.doi.org $/ 10.5888 /$ pcd 9.110241 .

Jung, M.-L., \& Loria, K. (2010). Acceptance of Swedish e-health services. Journal of Multidisciplinary Healthcare, 3, 55-63.

Linacre, J. M. (2009). Winsteps - Rasch Model computer program (Version 3.69.1.16).www.winsteps.com. Chicago, IL.

Linacre, J. M. (2004). Optimizing rating scale category effectiveness. In E.V. Smith and R.M. Smith (Eds.), Introduction to Rasch measurement (pp. 258-278). Maple Grove, MN: JAM Press.

McKhann, G., Drachman, D., Folstein, M., Katzman, R., Price, D., \& Stadlan, E.M. (1984). Clinical diagnosis of Alzheimer's disease: Report of NINCDS-ADRDA Work Group under the auspices of Department of Health and Human Task Force on Alzheimer's disease. Neurology, 34, 939-944. 
Manafo, E., \& Wong, S. (2012). Assessing the eHealth literacy skills of older adults: A preliminary study. Journal of Consumer Health on the Internet, 16, 369-381.

Nygård, L. (2008). Everyday Technology Use Questionnaire (ETUQ). Unpublished manual, research version 2. Karolinska Institutet, Stockholm, Sweden.

Nygård, L., Pantzar, M., Uppgard, B., \& Kottorp, A. (2011). Detection of disability in older adults with MCI or Alzheimer's disease through assessment of perceived difficulty in using everyday technology. Aging and Mental Health, 16, 361-371.

Pak, R., Price, M. M., \& Thatcher, J. (2009). Age-sensitive design of online health information: Comparative usability study. Journal of Medical Internet Research, 11. Doi: 10.2196/jmir.1220

Petersen, R. C. (2004). Mild cognitive impairment as a diagnostic entity. Journal of Internal Medicine, 256, 183-194.

Rosenberg, L., Kottorp, A., Winblad, B., \& Nygård, L. (2009a). Perceived difficulty in everyday technology use among older adults with or without cognitive deficits. Scandinavian Journal of Occupational Therapy, 16, 216-226.

Rosenberg, L., Nygård, L., \& Kottorp, A. (2009b). Everyday Technology Use Questionnaire (ETUQ) - psychometric evaluation of a new assessment of competence in technology use. OTJR: Occupation, Participation and Health, 29, 52-62.

Statistics Sweden (2013). Use of computers and the Internet by private persons in 2012. Statistiska centralbyrån.

Statistical Package for Social Sciences. (2011). Version 20. [Computer Software]. Chicago: SPSS Inc.

US Department of Health and Human Services (2006). Expanding the Reach and Impact of Consumer e-Health tools. http://www.health.gov/communication/ ehealth/ehealthtools/pdf/ehealthreport.pdf

Vanagas, G., \& Klimavičiūtè-Gudauskiene, R. (2012). Factors Affecting Electronic Health Information Needs in Primary Care Patients. Telemedicine and e-Health, 18, 724-728.

Van Deursen, A. J. A. M. (2012). Internet skill-related problems in accessing online health information. International Journal of Medical Informatics, 81, 61-72.

Van Deursen, A. J. A. M. \& Van Dijk, J. A. G. M. (2011). Internet skills performance test: Are people ready for eHealth? Journal of Medical Internet Research, 13:e35. DOI: 10.2196/jmir.1581.

Winblad, B., Palmer, K., Kivipelto, M., Jelic, V., Fratiglioni, L., Wahlund, L.-O., et al. (2004). Mild cognitive impairment - beyond controversies, toward a consensus: report of the International Working Group on Mild Cognitive Impairment. Journal of Internal Medicine, 256, 240-246. 